\title{
A nutrição na doença diverticular e na diverticulite em idosos: uma revisão
}

Jucimara Martins dos Santos,

Nutricionista do Centro do Idoso da Prefeitura Municipal de Barra Mansa, Professora Universitária do Centro Universitário de Barra Mansa (UBM) dos Cursos de Nutrição, Enfermagem, Fisioterapia e Educação Física. Mestre em Ciência pela UFRRJ.

ORCID: https://orcid.org/0000-0001-5121-6061

E-mail: jucimara martins@yahoo.com.br Angela Cristina dos Santos Ferreira,

Acadêmica do Curso de Nutrição UBM do Grupo de Estudos de Nutrição Clínica do Estágio em Nutrição Clínica ORCID: https://orcid.org/0000-0001-5112-2700

E-mail: angela-cristina-123@hotmail.com Diana Aparecida Ferreira de Souza, Acadêmica do Curso de Nutrição UBM do Grupo de Estudos de Nutrição Clínica do Estágio em Nutrição Clínica ORCID: https://orcid.org/0000-0001-6729-0362

E-mail: Di souza 2009@hotmail.com Suellen Alexandrino Ribeiro, Acadêmica do Curso de Nutrição UBM do Grupo de Estudos de Nutrição Clínica do Estágio em Nutrição Clínica ORCID: https://orcid.org/0000-0002-3734-1963

E-mail: suellenalexandrino@hotmail.com Regiane Izabel de Oliveira Dias. Acadêmica do Curso de Nutrição UBM do Grupo de Estudos de Nutrição Clínica do Estágio em Nutrição Clínica ORCID: https://orcid.org/0000-0001-8682-6943

E-mail: regianedias070@gmail.com

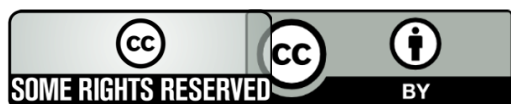




\section{Resumo}

Um indivíduo envelhece à medida que a sua idade aumenta. Este é um processo irreversível, natural e individual. É acompanhado por perdas progressivas de função e de papéis sociais. O surgimento da doença diverticular está relacionado com as alterações morfológicas e biomecânicas do colón, comprometendo a perda da elasticidade da parede colônica, atrofia mucos e aumento da pressão do colón. E essa alteração fisiológica associada a uma dieta pobre em fibras e em água acompanhada da inatividade física, uso de medicações e outros fatores podem desencadear a patologia diverticular. Uma vez instalada, a doença diverticular, também por diversos fatores, pode evoluir para seu quadro mais grave, a diverticulite. Esta revisão bibliográfica teve a intenção de trazer a relação da dieta e da qualidade de vida na prevenção e tratamento da doença diverticular e da diverticulite, sabendo que esta tem uma alta taxa de morbimortalidade. Conclui-se que é possível garantir a qualidade de vida do idoso, apesar das transformações morfológicas e estruturais, com uma dieta balanceada e adequada, além de um estilo de vida saudável com atividade física.

Palavras-chaves: envelhecimento, doença diverticular, diverticulite, nutrição

\section{Abstract}

An individual ages as his age increases. This is an irreversible, natural and individual process. It is accompanied by progressive loss of function and social roles. The appearance of diverticular disease is related to the morphological and biomechanical changes of the colon, compromising the loss of elasticity of the colonic wall, atrophy of mucus and increased pressure of the colon. And this physiological change associated with a diet low in fiber and in water, accompanied by physical inactivity, use of medications and other factors can trigger diverticular pathology. Once installed, diverticular disease, also due to several factors, can progress to its most serious condition, diverticulitis. This bibliographic review was intended to bring the relationship between diet and quality of life in the prevention and treatment of diverticular disease and diverticulitis, knowing that it has a high rate of morbidity and mortality. It is concluded that it is possible to guarantee the quality of life of the elderly, despite the morphological and structural changes, with a balanced and adequate diet, in addition to a healthy lifestyle with physical activity.

Keywords: aging, diverticular disease, diverticulitis, nutrition 


\section{Introdução}

Segundo a ONU (Organização das Nações Unidas) a idade de 65 anos é o ponto de corte entre o adulto e o idoso, porém nações desenvolvidas consideram 60 anos como ponto de corte (GALISA, et al, 2008).

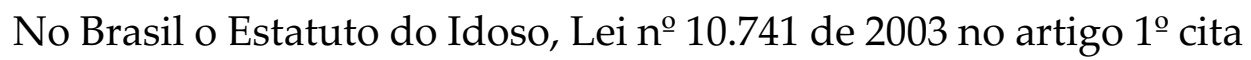
que idosos:

... são pessoas com idade igual ou superior a 60 anos".

O processo de envelhecimento é contínuo e se inicia com a concepção e só termina com a morte. Com o envelhecimento, o sistema digestivo apresenta alterações estruturais, de motilidade e da função excretora. Essas alterações variam em intensidade e natureza em cada segmento (GALISA, et al, 2008; FERRIOLLI in FREITAS et al, 2011). Várias são as perdas de funções decorrentes do declínio biológico, sendo comuns a diminuição da massa magra e constipação devido baixo consumo de frutas, verduras e legumes e diminuição da atividade física (GALISA et al, 2008).

Vários autores citam que são escassos e contraditórios os estudos acerca do envelhecimento sobre o intestino, tanto na morfologia como na estrutura. Em geral, os efeitos do envelhecimento sobre o intestino referem-se a aumento da prevalência de constipação intestinal, aumento da incidência de neoplasia e aumento da prevalência de doença diverticular ou diverticulose (FERRIOLLI in FREITAS et al, 2011).

A diverticulite, complicação da doença diverticular, (figura 1) teve maior incidência no século $X X$, sendo considerada no século XIX extremamente rara. Esse fato pode ter ocorrido devido a maior precisão do diagnóstico da doença, a maior parcela de idosos na população ou pela redução de fibras na dieta (CHEUCZUK et al, 2016; SILVA, 2015). A diverticulite caracteriza-se por dor 
abdominal aguda e constante, dor no quadrante inferior esquerdo, febre, leucocitose, podendo apresentar náusea, diarreia e constipação (MATOS, 2020).

Figura 1 - O cólon intestinal, divertículos, diverticulose (MATOS, 2020).

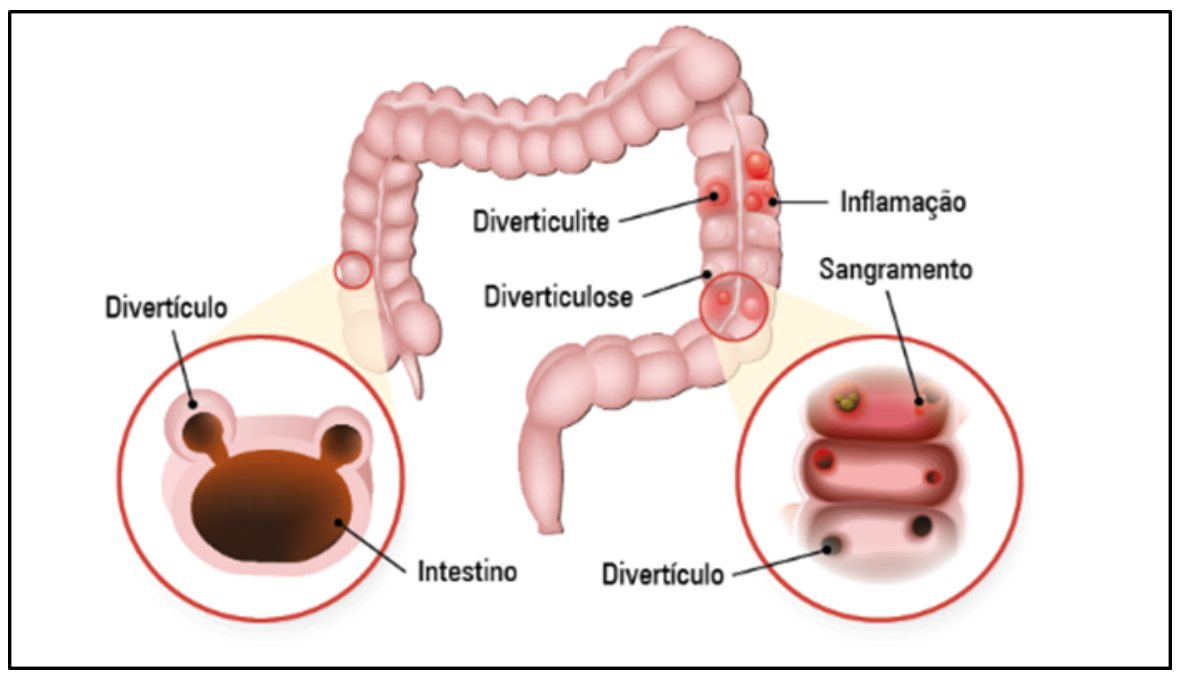

Sabe-se que o envelhecimento é um processo natural que pode refletir na mudanças fisiológicas do indivíduo e que ocorre diversas alterações no corpo e no organismo do idoso, desde a boca até o trato intestinal e gerando complicações como o aparecimento da doença diverticular que está relacionada com as alterações morfológicas e biomecânicas do colón, comprometendo a perda da elasticidade parede colônica e aumento da pressão do colón, portanto, as dietas pobres em fibras e outros fatores podem desencadear essa patologia. (FREITAS \& PY, 2013). É uma doença extremamente comum no mundo ocidental devido padrão alimentar com alto consumo de alimentos industrializados e baixo consumo de frutas (SILVA, 2015; MUGLIA, 2017).

Neste estudo, conduzido pela revisão bibliográfica de livros e de artigos científicos publicados na rede de dados, tem-se o objetivo de elucidar mais a relação da patologia com o processo de envelhecimento e o quanto a nutrição contribui na qualidade vida dos indivíduos idosos com doença diverticular. 


\section{Fundamentação Teórica}

\section{ETIOLOGIA E FISIOPATOLOGIA}

A doença diverticular está associada a formação de divertículos no colón intestinal, localizada maioritariamente no cólon sigmoide. São alterações decorrente da herniação das paredes da mucosa e da submucosa colônicas (figura 2) através dos músculos enfraquecidos e certo grau de atrofia da mucosa (JACOB FILHO \& KIKUCHI, 2011; MAHAN et al, 2018). Na maioria dos casos, é uma condição assintomática, mas também pode estar associada a sintomas e complicações, como hemorragias e diverticulite aguda (quando há sinais evidentes de inflamação) (SILVA, 2015; MAHAN et al, 2018).

Figura 2 - Fotografia de colonoscopia em paciente sem processo inflamatório de divertículos em cólon sigmoide (Imagem da Internet)

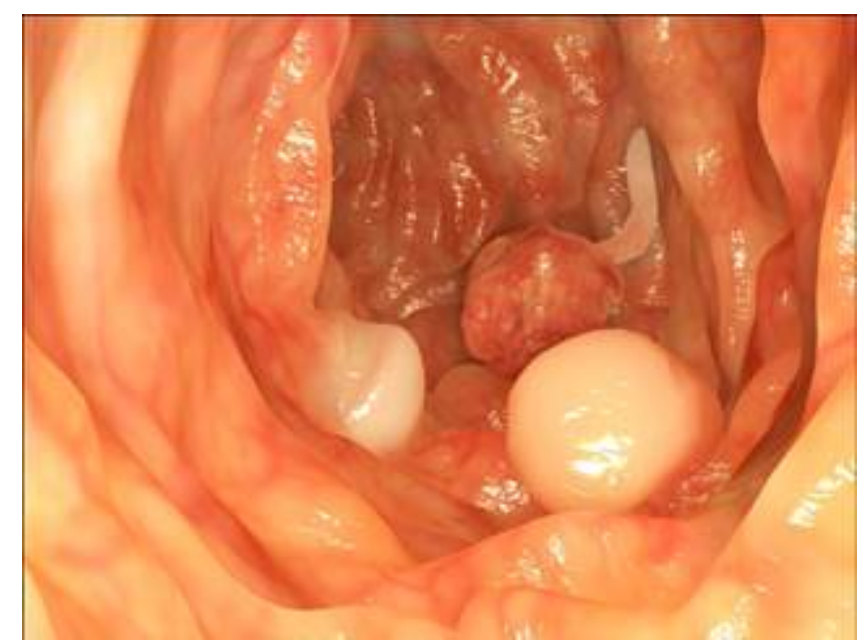

Inicialmente é necessário citar que, no idoso, a etiologia de qualquer doença é o próprio processo de envelhecimento, que pode aumentar o desenvolvimento e a frequência do surgimento de patologias, sendo a diverticulite uma dessas. No entanto, a idade em si não é um fator que aumenta 
a morbidade, mas sim a diminuição da capacidade funcional e das reservas funcionais provenientes da doença que resultam neste aumento (MARTINS et al.,2009 in CHEUCZUK; et al. 2016).

A etiologia da doença diverticular pode estar ligada à industrialização devido aos hábitos alimentares e à redução do consumo de fibras (SALLES, 2013), isso implica em uma dieta pobre em fibras, ocasionando constipação intestinal (fezes secas e duras). Na figura 3 segue um algoritmo de diagnóstico.

Figura 3 - Algoritmo da evolução da doença diverticular (MATOS, 2020)

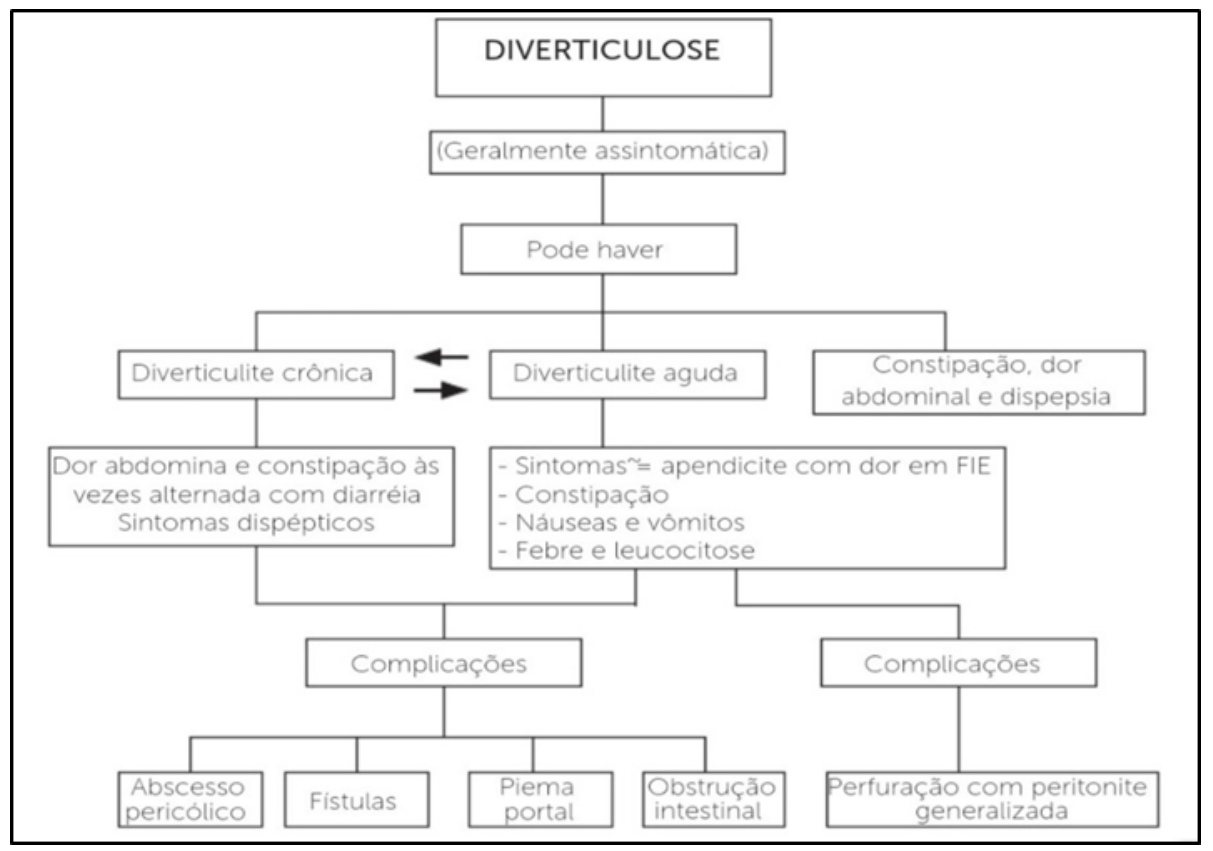

Embora a constipação tenha etiologia multifatorial extrínseca, uma das possíveis causas é este padrão alimentar, tendo também a inatividade física, uso de medicações, baixo consumo de água, como outros possíveis fatores etiológicos (FERRIOLLI in FREITAS et al, 2011; SILVA et al 2014; MAHAN et al, 2018). Quanto ao tempo de trânsito do bolo fecal não há um consenso se há ou não 
alteração do tempo de trânsito colônico, porém pode-se afirmar que a regulação deste trânsito em idosos seria a redução dos neurônios do plexo mioentérico associado ao envelhecimento (FERRIOLLI apud FREITAS et al, 2011).

A prevalência aumentada da doença diverticular em idosos está associado a alterações morfológicas e biomecânicas do cólon, com o comprometimento da resistência da parede colônica a pressões intraluminais elevadas (FERRIOLLI apud FREITAS et al, 2011; MAHAN et al, 2018). A presença de colágeno e elastina submucosos garante distensilidade ao cólon; alterações nesses elementos, com maior agregação e acumulação de colágeno e degeneração de fibrina podem causar menores distensilidade e resistência. Por outro lado, pode desconsiderarse que as alterações mencionadas não explicam integralmente a formação dos divertículos e que dietas pobres em fibras dietéticas, além de outros fatores extrínsecos, também apresentam importante papel na patogênese (FERRIOLLI apud FREITAS et al, 2011).A diverticulite é uma doença inflamatória benigna e considerada endêmica, além de apresentar boa resposta ao tratamento clínico e bom prognóstico para a maioria dos casos (figura 4).

Figura 4 - Diverticulite intestinal (imagem da internet)

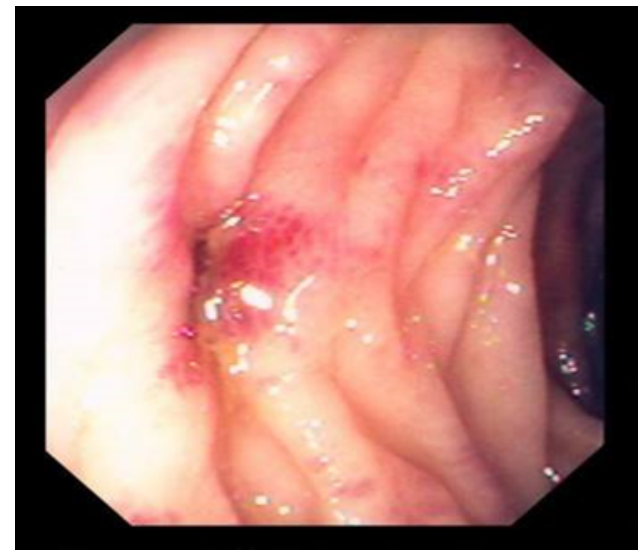

A diverticulite é caracterizada pela inflamação dos divertículos: uma massa dura pode ser formada no saco diverticular devido acúmulo de alimentos não digeridos e bactérias, o que pode ocasiona um bloqueio, o suprimento 
sanguíneo não atravessa as paredes finas do saco, seguido então de inflamação, perfuração ou hemorragia pela ação das bactérias presentes no cólon É considerada uma complicação da doença diverticular, geralmente atinge pessoas com mais de 70 anos, e também pode estar relacionada com obesidade, tabagismo, hipovitaminose D, sedentarismo (SILVA, 2015; MAHAN et al, 2018; CHEUCZUK et al, 2016).

A diverticulite pode evoluir para outras várias complicações, como formação de abscesso, fístula, perfuração com peritonite e estenose com obstrução colônica. A causa desse processo ainda não é bem compreendida. No entanto, o acúmulo de resíduos particulados no saco diverticular favorece a obstrução do colo estreito do pseudodivertículo e ocasiona supercrescimento bacteriano, isquemia tecidual local, inflamação e microperfurações. A inflamação é a causa principal das perfurações da parede dos divertículos (SALLES, 2013).

\section{MANIFESTAÇÃO CLÍNICA}

Na maioria dos pacientes a diverticulite é assintomática, permanecendo assim ao longo de suas vidas (SALLES, 2013; CHEUCZUK et al, 2016). Em geral os múltiplos divertículos, de 5 a $10 \mathrm{~mm}$ são mais encontrados no sigmóide (MATOS, 2020).

A diverticulite aguda é caracterizada por manifestações clínicas variadas na dependência da extensão do processo diverticular. A sintomatologia mais recorrente da doença é: cólica intermitente, constipação, distensão abdominal, desconforto no abdômen inferior e a sensação de um esvaziamento incompleto do reto. Algumas vezes, a constipação é alternante, ocorrendo também a diarreia. Pode-se citar também outros sintomas como: dor ou sensibilidade no quadrante esquerdo inferior, sangramento retal intermitente, febre, hemorragia 
microscópica ou intensa, calafrios, hipotensão e leucocitose (SALLES, 2013; CHEUCZUK et al, 2016).

A diverticulite pode evoluir para sua forma mais grave onde há sangramento, peritonite, hipoalbumineia, necessitando, muitas vezes, de hospitalização e cirurgia (MATOS, 2020).

\section{DIAGNÓSTICO}

Existe a possibilidade da doença diverticular ser diagnosticada pelos sintomas e sinais clínicos, porém, a acuidade diagnóstica do quadro clínico e exame físico é baixa. A avaliação do paciente com suspeita de doença diverticular ou diverticulite aguda deve ser baseada na anamnese e no exame físico detalhados inicialmente. É importante caracterizar a dor, definir sintomas associados e estar atento às evidências de possíveis complicações da doença (SALLES, 2013; MUGLIA, 2017).

Mas mesmo quando o diagnóstico clínico é bastante sugestivo, a extensão do processo inflamatório não é bem caracterizada clinicamente (MUGLIA, 2017). A avaliação complementar deve incluir hemograma completo e exame de urina rotina e o diagnóstico por imagem (SALLES, 2013). Desse modo, o diagnóstico por exames de imagens como a endoscopia, tomografia e colonoscopia se mostram muito importante. Uma série de estudos recentes na literatura radiológica brasileira tem demonstrado a importância da tomografia computadorizada (TC) na avaliação das doenças dos cólons ((SALLES, 2013; SEICHAS, 2019). A tomografia computorizada abdominal (TC) tem documentadas sensibilidade e especificidade próximas dos 100\% no diagnóstico de DAC, motivo pelo qual é considerada atualmente o exame gold standard para o diagnóstico e caracterização desta patologia (MELO et al, 2014). 
A colonoscopia é normalmente evitada em doentes com diverticulite aguda, pois, a inflação do ar e manipulação instrumental são considerados de alto risco de perfuração. Quando efetuada é necessário ser cuidadoso na manipulação e deverá minimizar a inflação de ar e se o diagnóstico de diverticulite aguda se confirmar deverá ser parada imediatamente (MELO et al, 2014).

A gravidade clínica da diverticulite associada a abscessos da doença pode ser realizada segundo classificação de Hinchey conforme Tabela 1 . O risco de morte é inferior a cinco, aproximadamente 13 e 43\% para pacientes nos estádios de Hinchey 1 e 2, 3 e 4, respectivamente (SALLES, 2013).

Tabela 1 - Classificação de Hinckey (1978)

\begin{tabular}{|c|c|}
\hline Estádio 1 & Abscesso paracólico pequeno, confinado ao mesentério do cólon \\
\hline Estádio 2 & Abscesso grande, distante, localizado na pelve ou retroperitônio \\
\hline Estádio 3 & Peritonite purulenta decorrente de ruptura de abscesso \\
\hline Estádio 4 & $\begin{array}{r}\text { Peritonite fecal decorrente de perfuração livre } \\
\text { de um divertículo não inflamado }\end{array}$ \\
\hline
\end{tabular}

Fonte: Salles (2013)

\section{TRATAMENTO}

O tratamento da doença diverticular varia dependendo da severidade dos sintomas, e é centrado em diminuir os sintomas e prevenir complicações. A diverticulite aguda apresenta-se de forma leve na maioria dos casos. Esta forma é tratada em ambiente ambulatorial sendo habitualmente eficaz e menos de $10 \%$ retornam com aumento da gravidade. O paciente é orientado com dieta específica para repouso intestinal e antibióticos por 7 a 10 dias (MAHAN et al, 2018; SEIXAS, 2019). E posterior dieta para prevenção de novo quadro agudo (LAJOLO \& PFRIMER, 2016; MAHAN et al, 2018). 
$\mathrm{Na}$ diverticulite complicada, estadiamento 3 a 4 , pode haver indicação cirúrgica e a mortalidade nessa eventualidade é elevada (SALLES, 2013; SEIXAS, 2019).

\section{DIETOTERAPIA NA DOENÇA DIVERTICULAR E NA DIVERTICULITE}

A população idosa mundial vem sofrendo rápido crescimento e essa longevidade leva a maior preocupação com relação ao estado nutricional e desenvolvimento de doenças. Neste tópico será tratado da dietoterapia para o paciente com doença diverticular ou com sua forma mais inflamada, a diverticulite.

Como dito, a presença de divertículos intestinais pode ser comum na população idosa, então, garantir uma alimentação saudável, com manutenção das reservas nutricionais e evitando doenças carenciais é a meta. Silva (2015) cita que os idosos representam um grupo vulnerável a carências alimentares diversas e sua alimentação pode tanto influenciar na etiologia de doenças como sua gravidade. E como várias alterações fisiológicas acontecerão com a progressão dos anos, nos intestinos é confirmado decréscimo do número de células absortivas intestinais e da área de superfície de absorção e diminuição do tônus muscular do cólon intestinal, com consequente constipação (SILVA et al, 2014).

A dieta, fator etiológico da diverticulite, está também relacionada aos alimentos ultraprocessados, ricos em conservantes, corantes e estabilizantes químicos. Os alimentos submetidos a transformações na indústria associado à mudança de hábitos alimentares, com a transição nutricional, fazem parte desta situação, além, dos distúrbios funcionais causados pela massa residual ingerida (CHEUCZUK et al. 2016).

A diminuição da percepção anorretal também pode agravar a constipação. Não raramente, os idosos também podem apresentar diarreia, causada por 
hipercrescimento bacteriano associado a má nutrição, diverticulite e diabetes. Alimentos que devem ser incluídos na alimentação diária: frutas, como laranja com bagaço, mamão, ameixa, abacaxi, cereais integrais, farelos de trigo e aveia. Também é importante aumentar a oferta hídrica, para que as fibras possam agir. Recomenda-se pelo menos 8 copos de água por dia. (SILVA et al, 2014).

No hospital, a diverticulite grave exige repouso intestinal total, ingestão oral geralmente é reduzida até que os sintomas diminuam e apenas pequena quantidade de água ou gelo picado poderá ser oferecido além da medicação e, dependendo do estado nutricional do paciente, de suporte de nutrição parenteral (NP). À medida que há regressão da inflamação, a dieta progride para líquida restrita. Na maioria dos casos, a antibioticoterapia e o repouso intestinal atenuam os sintomas dentro de 2 a 5 dias. E, assim, a dieta poderá evoluir para outra consistência de dieta, com exclusão de sementes oleaginosas, sementes ou vegetais fibrosos. A dieta terá restrição de fibras por até 1 mês, geralmente é prudente começar uma dieta pobre em fibras (10 a $15 \mathrm{~g} /$ dia). E, em seguida, com a melhoria do quadro clínico, a ingestão de fibras é liberada (WIDTH \& REINHARD, 2011; MAHAN et al, 2018).

Para indivíduos assintomáticos é importante ressaltar recomendação de um estilo de vida saudável, como praticar regularmente atividades físicas e uma dieta rica em fibras, com hortaliças e frutas e água (CHEUCZUK et al. 2016; MATOS, 2020).

Como o risco de recidiva de diverticulite aguda é superior a 18\% em 10 anos (SEIXAS, 2019), a seguir serão citados pontos importantes para a alimentação do idoso que desenvolveu doença diverticular.

\section{FIBRA DIETÉTICA}

A fibra da dieta é definida como a parte não digerível do alimento vegetal a qual resiste à digestão e absorção intestinal, porém, com fermentação completa 
ou parcial no intestino grosso. Pode ser classificada em fibra dietética solúvel e insolúvel. As fibras solúveis incluem as pectinas, gomas, mucilagens como psillium, um polissacarídeo viscoso. Entre as fibras insolúveis estão à celulose, a hemicelulose e a lignina (MONTES \& NASCIMENTO, 2013).

A FAO/OMS (2019) para pessoas acima de 50 anos recomenda o consumo de no mínimo $21 \mathrm{~g} /$ dia para gênero feminino e $30 \mathrm{~g} /$ dia para gênero masculino. Essa recomendação de consumo de fibras na dieta tem a finalidade de auxiliar na prevenção do aparecimento de doenças crônicas relacionadas à dieta (MONTES \& NASCIMENTO, 2013). Comprovando essa indicação nutricional, a redução do bolo fecal é decorrente de uma alimentação pobre em fibras dietéticas, principalmente as insolúveis, acarretando na diminuição da motilidade colônica, ressecamento das fezes, e da secreção de muco e possível crescimento bacteriano, o que leva ao aumento da pressão intraluminal e, possivelmente, herniação da mucosa, chamados de divertículos (AFONSO et al.,2011 apud CHEUCZUK et al. 2016; MAHAN et al, 2018, MATOS, 2020).

O consumo de fibra dietética solúvel e insolúvel está associada com a diminuição dos riscos da diverticulite. Uma revisão realizada sobre os fatores dietéticos associados com a doença indicou que uma dieta rica em fibras e pobre em gorduras e carne vermelha diminui os riscos de diverticulite, porém não há dados sobre a quantidade consumida desses dois alimentos (TURSI \& DANESE, 2015 apud CHEUCZUK et al. 2016)

Assim que o episódio agudo se resolve, é importante iniciar uma dieta rica em fibras para otimizar os movimentos intestinas e tentar diminuir a consistência das fezes (MAHAN et al, 2018; SEIXAS, 2019; MATOS, 2020). As fibras alimentares são capazes de armazenar água, aumentar a massa luminal e diminuir a pressão intestinal, podendo assim, reduzir os sintomas e melhorar a função do intestino (SEIXAS, 2019). 
A ingestão de fibras deve aumentar gradualmente porque pode causar distensão abdominal devido quadro de flatulência. Se um paciente não puder ou não quiser consumir a quantidade necessária de fibras, suplementos de metilcelulose ou fibras de Psyllium podem ser utilizados com bons resultados. Uma dieta rica em fibras, possivelmente com suplementação de fibras, é preconizada na diverticulose assintomática para reduzir a probabilidade de progressão da doença, impedir a recorrência de episódios de sintomas e prevenir diverticulite aguda. (MAHAN et al, 2018).

Uma dieta rica em fibras combinada a uma hidratação adequada promove fezes pastosas e volumosas que são eliminadas mais rapidamente e precisam de menos esforço na defecação (MAHAN et al, 2018).

\section{ÁGUA}

A proporção de água no organismo humano varia nas diversas faixas etária, diminuindo com o avançar da idade. Com o envelhecimento ocorre uma redução de 0,3L/ano desde a idade adulta até aproximadamente 70 anos. A partir desta idade a diminuição é mais acentuada, estando acentuada a perda da massa magra (ANICETO et al, 2015).

A capacidade de absorção de eletrólitos, provenientes da ingestão hídrica também pode ser alterada pela diverticulite. Torna-se difícil prever quanto de água é necessário para a adequada dietoterapia nessa doença, portanto recomenda-se $35 \mathrm{~mL} / \mathrm{kg}$ de peso atual/dia (CHEUCZUK et al, 2016).

\section{PROBIÓTICOS}

O uso e probióticos tem sido proposto por vários investigadores, com vista a alterar a microbiota do cólon e influenciar a fisiopatologia da doença diverticular. Essa indicação terapêutica se dá justamente por saber-se que em um 
indivíduo com microbiota intestinal alterada, observa-se maior vulnerabilidade à infecções (SANTOS \& SÁ, 2007; LAJOLO \& PFRIMER, 2016; SEIXAS, 2019).

Poli (2016) apud Matos (2020) afirma que o uso de probióticos associado ou não a mesalazina (antiinflamatório) é útil na prevenção de doença diverticular assintomática. Na Tabela 2 segue uma sugestão com probióticos e seus gêneros.

Tabela 2- Principais probióticos utilizados para manutenção da microbiota intestinal.

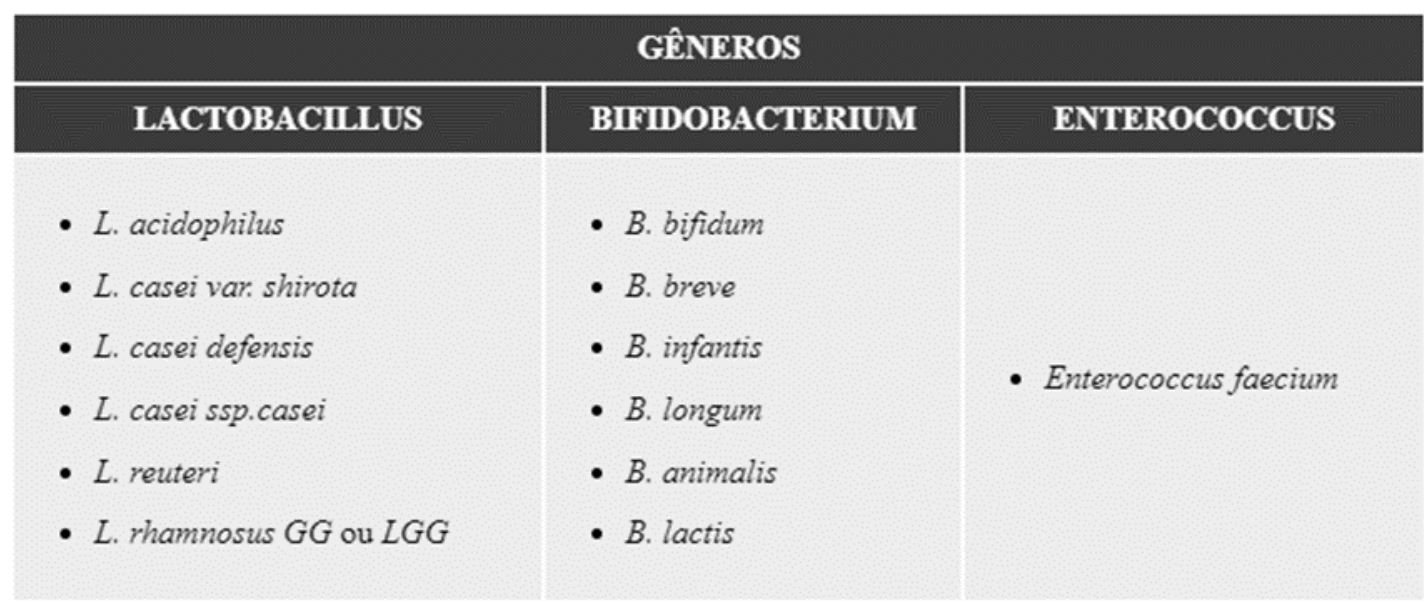

Fonte: Matos, 2020.

\section{PROTEÍNA}

Uma ingestão adequada de macro e micronutrientes deve ser considerada como requisito essencial para qualquer método terapêutico bem-sucedido em idosos (FREITAS et al, 2015). Um dos maiores riscos de perda de peso no envelhecimento, que pode ser multifatorial, é o desenvolvimento da sarcopenia e da caquexia. Todos os idosos devem consumir pelo menos 0,8g/kg/dia de proteínas para garantir uma síntese proteica adequada nos músculos. Entretanto, situações de vulnerabilidade que são muito comuns em idosos, que variam desde emocionais, físicas, patológicas ou circunstanciais, fazem ser necessário aumentar o aporte proteico deste grupo populacional (FREITAS et al, 2015; LAJOLO \& PFRIMER, 2016). 
Todas estas condições de vulnerabilidade têm implicações nas recomendações proteicas dos idosos. As recomendações proteicas para idosos e adultos saudáveis pelas DRIs é de 0,8-1,0 g/kg de peso/dia - Recommended Dietary Allowance (RDA), e a variação mínima avaliada pela Estimated Average Requirements (EAR) é de 0,66 g/kg de peso/dia. Vale destacar que este valor é considerado de proteínas de alto valor biológico e não considera o nível de atividade física (LAJOLO \& PFRIMER, 2016, MAHAN et al, 2018).

Em caso de os idosos com alguma doença aguda ou crônica, a necessidade pode ser de 1,2 a 1,5 g/kg de peso/dia, e ainda se esta doença for grave e juntamente com a desnutrição, normalmente presente em idoso, podem-se atingir até 2,0g/kg de peso/dia (LAJOLO \& PFRIMER, 2016).

É importante também a escolha dos alimentos proteicos, pois as proteínas que são digeridas rapidamente (por exemplo, a proteína de soro de leite) induzem a uma melhor resposta anabólica em pessoas com mais idade do que as proteínas que são digeridas lentamente (por exemplo, a caseína) (FREITAS et al, 2015).

\section{DOENÇA DIVERTICULAR E ABSORÇÃO DE NUTRIENTES}

Modificações intestinais são observadas no indivíduo idoso. Ocorre certo grau de atrofia na mucosa e no revestimento muscular, o que resulta na deficiência de absorção de nutrientes (JACOB FILHO \& KIKUCHI, 2011). Com as alterações intestinais em indivíduos da terceira idade, ocorre a atrofia na mucosa e na musculatura intestinal, que resulta na deficiência e má absorção de nutrientes. (GAVANSKI al et, 2015). É importante considerar que mesmo pequenas alterações fisiológicas na digestão e absorção de nutrientes, juntamente à motilidade alterada, contribuem para o desenvolvimento de desnutrição em idosos, em médio e longo prazos. O uso de diversos medicamentos 
(polifarmácia), tão comum neste grupo etário, também interfere negativamente no estado nutricional (SILVA et al, 2014).

Durante o envelhecimento, o campo de absorção do intestino delgado vai diminuindo com a redução da altura das vilosidades, o que resulta em menor absorção de alguns micronutrientes, como o cálcio e o ferro. Queixas clínicas como constipação e fecaloma são comuns nesse período, aumentando também a incidência de diverticulose (FIDELIX et al, 2013).

\section{ORIENTAÇÃO NUTRICIONAL PARA IDOSO COM DIVERTICULITE}

Como comprovado anteriormente, é necessário que o idoso tenha uma alimentação saudável para se manter mais fortalecido na prevenção de doenças. O Ministério da Saúde (BRASIL, 2009) através dos 10 passos da alimentação do idoso, faz uma orientação de como deve ser esta alimentação saudável:

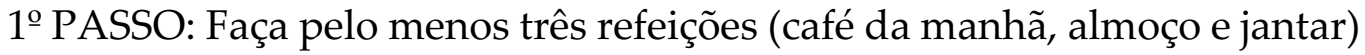
e dois lanches saudáveis por dia. Não pule as refeições!

2 PASSO: Inclua diariamente seis porções do grupo dos cereais (arroz, milho, trigo, pães e massas), tubérculos como a batata, raízes como mandioca/ macaxeira/ aipim, nas refeições. Dê preferência aos grãos integrais e aos alimentos na sua forma mais natural.

3o PASSO: Coma diariamente pelo menos três porções de legumes e verduras como parte das refeições e três porções ou mais de frutas nas sobremesas e lanches.

4⿳⺈ PASSO: Coma feijão com arroz todos os dias ou, pelo menos, cinco vezes por semana. esse prato brasileiro é uma combinação completa de proteínas e bom para a saúde. 
5ำ PASSO: Consuma diariamente três porções de leite e derivados e uma porção de carnes, aves, peixes ou ovos. retirar a gordura aparente das carnes e a pele das aves antes da preparação torna esses alimentos mais saudáveis!

6ํㅗ PASSO: Consuma, no máximo, uma porção por dia de óleos vegetais, azeite, manteiga ou margarina.

$7^{\text {o }}$ PASSO: Evite refrigerantes e sucos industrializados, bolos, biscoitos doces e recheados, sobremesas doces e outras guloseimas como regra da alimentação. coma-os, no máximo, duas vezes por semana.

8o PASSO: Diminua a quantidade de sal na comida e retire o saleiro da mesa.

9o PASSO: Beba pelo menos dois litros (seis a oito copos) de água por dia. Dê preferência ao consumo de água nos intervalos das refeições.

10 PASSO: Torne sua vida mais saudável. pratique pelo menos 30 minutos de atividade física todos os dias e evite as bebidas alcoólicas e o fumo (BRASIL, 2009).

\section{ORIENTAÇÃO NUTRICIONAL PARA DOENÇA DIVERTICULAR}

Depois da regressão da diverticulite grave, mas ainda portador da doença diverticular, alguns cuidados nutricionais devem ser realizados para manter o paciente estável e sem risco de agravamento, como pode ser visto no quadro 1:

Quadro 1 - Orientação nutricional para o paciente com doença diverticular

1 - Consumir diariamente de 6 a 11 porções de pão integral, cereais, farinhas e outros produtos à base de grãos integrais.

2 - Consumir de 5 a 8 porções de frutas e vegetais, principalmente legumes, frutas cruas com casca, legumes crus (cenoura, beterraba, aipo) e vegetais cozidos com casca (batata). 3 - Consumir diariamente $25 \mathrm{~g}$ de fibras. 
4 - Consumir 2litros de água por dia (8 copos de 250mL).

5 - Reduzir a ingestão de gorduras.

Fonte: Width \& Reinhard, 2011

\section{ATIVIDADE FÍSICA}

Um dos fatores para a longevidade é a manutenção da mobilidade, como os hábitos de vida saudável e o envelhecimento ativo, que são determinantes para a saúde do idoso. Manter a capacidade funcional adequada é o diferencial para o envelhecimento bem-sucedido (LAJOLO \& PFRIMER, 2016).

A constipação pode ser reduzida aumentando a ingestão de fibras, líquidos e também, pelo aumento de atividade física (MAHAN et al, 2018).

\section{MÉTODOS}

A pesquisa bibliográfica foi realizada por meio de consulta à base de dados Scientific Eletronic Library Online (SciELO) e PubMed. Foram utilizados artigos de revisão e originais, em língua portuguesa e inglesa, após a análise do título, resumo e textos completos.

Utilizaram-se os seguintes descritores: obesity, inflammation e Functional foods e todas as combinações de associação entre elas.

\section{Considerações Finais}

Se o processo de envelhecimento é um fato, garantir uma qualidade de vida deve ser a meta. É fato que um estilo de vida saudável influencia na qualidade do envelhecimento e tem feito com que, diariamente, mais pessoas adiram a práticas mais saudável como o exercício físico e a melhor qualidade da dieta.

É importante garantir uma alimentação rica em fibras e boa hidratação para que a doença diverticular não se instale. E caso haja o surgimento da doença, 
que esta não evolua para seu quadro mais grave. O idoso precisa ser atendido por uma equipe multiprofissional que possa ajudá-lo a diminuir os riscos nutricionais próprios da idade como também da patologia.

\section{Referências}

AFONSO, M.; PINTO, J.; VELOSO, R.; FREITAS, T.; CARVALHO, J.; FRAGA, J. Visceral fat: A key factor in diverticular disease of the colon. Portuguese Journal of Gastroenterology, v. 19, n. 2, p.62-65, jul.2011. Disponível em: www.scielo.com Acessado em 20/04/2020.

ANICERTO, A; FERREIRA, D.C.F.; BARBOSA, M.; DIAS, S.; XARÁ, S. RODRIGUES, T. Hidratação no Ciclio da Vida. Ebook. Associação Portuguesa de Nutricionistas - APN. Março. N.36, 2015.

BRASIL. Ministério da Saúde. Secretaria de Atenção à Saúde. Departamento de Atenção Básica. Alimentação saudável para a pessoa idosa: um manual para profissionais de saúde / Ministério da saúde, Secretaria de Atenção à Saúde, Departamento de Atenção Básica. - Brasília: Editora do Ministério da Saúde, 2009. 36p. - (Série A. Normas e Manuais Técnicos).

CHEUCZUK, E. C.; COSTA, E. C.; REBEQUI, F.; GÓES, G. DE O.; MAZUR, C. E. A DIETOTERAPIA COMO TRATAMENTO FUNDAMENTAL PARA DIVERTICULITE. Biológicas \& Saúde, v. 6, n. 22, 18 nov. 2016. Disponível em: https://ojs3.perspectivasonline.com.br/biologicas e saude/article/view/1051/805 Acessado em: 12/05/2020.

FIDELIX, M.S.P; SANTANA, A.F.F; GOMES, J.R. Prevalência de desnutrição em idosos. RASBRAN - Revista da Associação Brasileira de Nutrição. São Paulo, SP, Ano 5, n. 1, p. 60-68, Jan-Jun. 2013. Disponível em: $\underline{\text { www.scielo.com }}$ Acessado em 20/04/2020. 
FREITAS A.F.; PRADO, M.A.; CAÇÃO, J.C.D.; ALBERTINI, B.S.. Sarcopenia e estado nutricional de idosos: uma revisão da Literatura. Arq. Ciênc. Saúde. 2015 jan-mar; 22(1) 09-13. Disponível em:

http://www.cienciasdasaude.famerp.br/index.php/racs/article/view/19/10 Acessado em: 20/04/2010.

FREITAS, E.V.; PY , L.. Tratado de geriatria e gerontologia. $3^{\circ}$ Ed. Rio de janeiro: Guanabara Koonga, 2013. 1639 p.

GALISA, M.S.; ESPERANÇA, L.M.B.; SÁ, N.G. Nutrição - conceitos e aplicações. RJ: MBooks, 2008.

GAVANSKI, D.S; BARATTO, I; GATTI, R.R. Avaliação do hábito intestinal e ingestão de fibras alimentares em população de idoso. Revista Brasileira de Obesidade, Nutrição e Emagrecimento, São Paulo. v.9. n.49. p.3-11. Jan./Fev. 2015. Disponível em: www.scielo.com Acessado em 22/04/2020.

JACOB FILHO, W.; KIKUCHI, E.L. Geriatria e Gerontologia Básica. $1^{\circ}$ ed. Rio de Janeiro:Elsevier, 2011.

LAJOLO, F.M.; PFRIMER, K. Nutrição e Envelhecimento Saudável. SP: ILSI Brasil_international Life Sciences Institute Brasil, 2016.

MAHAN, L.K.; ESCOTT-STUMP, S.; RAYMOND, J.L. Krause: Alimentos, Nutrição e Dietoterapia. 14ª ed. Rio de Janeiro: Elsevier, 2018. 4247 p.

MARTINS, J.F.; ROCHA, J.G.; MIRANDA, E.F.; SARTOR, M.C.; STECKERT, J.S.; STECKERT, A.S.; GUIMARÃES, P.R.B.; KOTZE, P.G. Análise da prevalência de entidades coloproctológicas nos pacientes idosos do serviço de coloproctologia de um hospital universitário. Revista brasileira de Coloproctologia, v.29, n. 2, p. 145-157, 2009. Disponível em: www.scielo.com Acessado em 20/04/2020.

MATOS, L.A. Dietoterapia das doenças gastrointestinais e glândulas anexas. SP: Editora SENAC, 2020.

MELO, F.; LOPES, L.; OLIVA, A.; BERNARDES, A.; OLIVEIRA, F.J.

Diverticulite aguda cólica: qual o valor da ecografia abdominal? Rev. Port. 
Cir. no.29 Lisboa jun. 2014 Disponível em

http://www.scielo.mec.pt/scielo.php?script=sci arttext\&pid=S164669182014000200004 Acessado em 12/05/2020.

MONTE F.V.; NASCIMENTO, K.O. Associação do consumo do amido resistente na prevenção e tratamento do diabetes mellitus tipo 2. Revista Verde (Mossoró - RN - BRASIL), v. 8, n. 5, p. 12 - 19, (Edição Especial) dezembro, 2013. Disponível em

https://editoraverde.org/gvaa.com.br/revista/index.php/RVADS/article/view/19 51/2174 Acessado em 11/05/2020.

MUGLIA, V. F. Doença diverticular dos cólons: evolução da abordagem terapêutica e papel da tomografia computadorizada nos quadros agudos. Radiol Bras. 2017 Mar/Abr;50(2):IX-X. Disponível em https://www.scielo.br/pdf/rb/v50n2/pt 0100-3984-rb-50-02-00IX.pdf Acessado em 12/05/2020.

SALLES, R.L.A. Doença diverticular dos cólons e diverticulite aguda: o que o clínico deve saber. Revista Medicina de Minas Gerais 2013; 23(4): 490-496 Disponível em file://C:/Users/jucim/Downloads/v23n4a10\%20(4).pdf. Acessado em $10 / 05 / 2020$

SANTOS, J.M. SÁ, L.C. Probiótico: iogurte e leite fermentado no controle da enterocolite associada a antibióticos. Revista Científica do Centro

Universitário de Barra Mansa - UBM. v.9, n. 17, jul. 2007. p. 28-34 .

SEIXAS, P.P. A abordagem da diverticulite aguda: perspetivas atuais e futuras.

Dissertação de Mestrado Universidade a Beira Interior. Ciências da Saúde. Covilhã. 2019. Disponível em

https://ubibliorum.ubi.pt/bitstream/10400.6/8695/1/6962 14776.pdf Acessado em 03/05/2020.

SILVA, L.M.S. Doença diverticular do cólon no idoso. Tese de Mestrado FMUC Medicina 2015 Disponível em https://estudogeral.sib.uc.pt/bitstream/10316/30793/1/TESEMESTRADO.pdf Acessado em 05/05/2020.

SILVA, M.L.N; MARUCCI, M.F.N.; ROEDIGER, M.A.(orgs) Tratado de Nutrição em Gerontologia. SP: Editora Manole, 2014. 
SANTOS, J. M. ; FERREIRA, A. C. S. ; SOUZA, D. A. F. S. ;RIBEIRO, S. A. R. ; DIAS, R. I. O. A nutrição na doença diverticular e na diverticulite em idosos: uma revisão. R. Científica UBM - Barra Mansa (RJ), ano XXV, v. 22, n. 42,1 . Sem. 2020 p. 116-137 ISSN 1516-4071

WIDTH, M.; REINHARD, T. MdS, Manual de Sobrevivência para Nutrição

Clínica. Trad. CONSENDEY, Carlos Henrique. RJ: Guanabara Koogan, 2011. 